\title{
A Study of Compaction Densification Behavior of Composite Particles by Multiparticle Finite Element Method
}

\author{
Junnan Li, ${ }^{1}$ Peng Han $\mathbb{D}^{1},{ }^{1}$ Yingshi Xu, ${ }^{2}$ Kun Liu, ${ }^{1}$ Guoxin Zhang, ${ }^{1}$ and Peng Xu ${ }^{1}$ \\ ${ }^{1}$ School of Materials and Metallurgy, University of Science and Technology Liaoning, Anshan, Liaoning 114000, China \\ ${ }^{2}$ Angang Steel Corporation Limited, Anshan, Liaoning 114000, China \\ Correspondence should be addressed to Peng Han; hanpeng@ustl.edu.cn
}

Received 21 May 2021; Accepted 18 January 2022; Published 10 February 2022

Academic Editor: AMA Neves

Copyright (c) 2022 Junnan Li et al. This is an open access article distributed under the Creative Commons Attribution License, which permits unrestricted use, distribution, and reproduction in any medium, provided the original work is properly cited.

In this paper, 3D particulate scale modelling on the die compaction of DEM generated initial packings of both soft and hard particles was conducted by employing the multiparticle finite element method (MPFEM). The effects of initial packing structures as well as the compaction pressure on the macroscopic and microscopic properties of the whole powder mass and local structures were investigated. In addition, corresponding physical experiments were carried out for model validation. The results show that the compact obtained from the initial dense packing under vibration undergoes yielding stage earlier than that with natural initial packing (without vibration), and the relative density is much higher. Pores that are significantly smaller and with more uniform size and homogenous stress distribution are observed in the former case. Highest stress regions occur in most cases at a grain boundary with large curvature after deformation. Moreover, the high stress in the central part of both soft and hard particles during compaction is significantly reduced after pressure unloading, reaching a new force balance. In this case, the stress is concentrated mainly at the corners of the deformed particles, which creates the risk of cracking during subsequent sintering at either the contact region between particles or the corners. The numerical results are found to be in good agreement with those from physical experiments, confirming the robustness and reliability of the numerical model used in the simulations.

\section{Introduction}

Powder metallurgy (PM) has long been an indispensable technology for both advanced and conventional materials [1-4]. Its advantages lie in cost-effectiveness, straightforward operation, conservation of materials, environmental friendliness, high production rates, and net or near net shape forming for complex geometrical and structural metals or alloys with high melting point or porous structure. These properties make it very competitive with conventional metallurgical processes such as casting or other processes such as forging, machining, and others $[5,6]$. An important part of the preparation of PM for these materials is powder forming, which has a large impact on the subsequent sintering process and on the performance of the final PM product. For this reason, a great deal of research attention, both experimental and theoretical, has been focused on this in the previous decades.
Regarding physical experiments, most of the focus is on establishing the relationship between the relative density of the compaction and the external pressure during the powder forming process. Since Walker first proposed the linear relationship between the relative volume of the powder and the logarithm of the compaction pressure based on experiments, various formulas have been proposed to predict the quantitative relationship between compaction pressure and relative density. However, it proved difficult to quantitatively characterize the local density distribution, stress distribution, and particle flow evolution process of the green body in the powder forming process with physical experiments, due to the geometric, material, and contact nonlinearities [7-11]. In this context, various numerical models describing the forming of metal powders have been proposed. From the perspective of metal plastic mechanics, based on the von Mises theory of metal materials, Kuhn [12], Green [13], Shima [14], Gurson [15], Doraivelu [16], and others have 
proposed different forms of yield criteria for powder materials. For soil plastic mechanics methods, Drucker-PragerCap model [17] and Cam-Clay model [18] are mainly represented. Based on these yield models, a macrocontinuous finite element method (FEM) was developed to solve the difficult problems encountered in physical experiments. The main advantage of the macroscopic continuous scale finite element method is that it can be used to analyze the macroscopic characteristics of the local density and distribution, the stress distribution, and the particle displacement during the forming process. In previous research, this method was successfully used for unidirectional molding of pure metal powder [19-21] and composite powder $[22,23]$. Although the traditional finite element method can avoid the difficulties present in physical experiments to a certain extent, the powder particles are regarded as a continuum with uniform distribution in the FEM model, so it is difficult to characterize the particle dynamics and contact mechanical behavior in the powder forming process from the microscale. The deficiency of FEM simulation in this aspect can be compensated with a discrete element (DEM) simulation. Using this method, An et al. [24-27] successfully simulate the packing densification of spherical and nonspherical particles. However, the study found that the limitation of DEM is that it is only suitable for the simulation of small deformation particle accumulation, and it is difficult to simulate the formation of particles with large plastic deformation; in particular when the packing density of particles exceeds 0.85 , it is very difficult to implement DEM [28]. Moreover, since it can amend the drawbacks presented by both the FEM and DEM simulation, the multiparticle finite element method (MPFEM) proposed in recent years has been gaining currency [29-43]. However, few studies on the simulation of soft and hard particles forming process from the particle scale using multiparticle finite element (MPFEM) can be found in literature. Only Wu et al. [40] simulated the $2 \mathrm{D}$ forming process of composite powder, and only the formation of ordered initial packing structure was considered. In fact, the initial packing of powder before forming is usually disordered, and the research in this aspect is only considered in our previous $2 \mathrm{D}$ simulation of composite powder [44, 45]. However, the simulation of $2 \mathrm{D}$ forming process is not sufficient to describe the evolution of compact structure and properties as well as the micromechanism of densification during 3D powder forming, and the research is not presently available.

For this reason, in this paper, MPFEM was used to simulate the compaction process of soft and hard particles on a 3D particle scale. The influence of powder density and initial packing structure was considered throughout the entire process. The density and distribution of compacts, stress and distribution, morphology evolution of particles, filling mechanism of pores, stress transfer behavior and variation of internal stress of different particles during forming, and pressure relief of soft and hard particles were systematically analyzed. The results are found to be of great significance for the preparation of high-performance soft and hard particles compacts and the analysis of the densification mechanism.

\section{Simulation Method and Conditions}

2.1. Model Setup. The particle scale MPFEM numerical simulation was carried out using commercial MSC Marc software. In order to reduce constraints and obtain a better simulation of the physical process, DEM dynamic modelling was employed to generate initial random packing structure which was then imported into the FEM model for compaction. The DEM method has been successfully applied to packing of various spherical and nonspherical particulate systems [24-27]. Figure 1(a) shows the DEM generation of initial random packing structure with discretized mesh division on all particles before compaction when the mass fraction of soft particles is $X_{\text {soft }}=25 \mathrm{wt}$.\%, where each particle includes 1014 units and 872 nodes as shown in Figure 1(b). In this case, both soft and hard particles have equal size ( $1 \mathrm{~mm}$ in diameter). At the interface between two particles in contact, the Coulomb friction model was used:

$$
\sigma_{\mathrm{fr}}=-\mu \sigma_{n_{\pi}} \frac{2}{\pi} \arctan \left(\frac{v_{r}}{r_{v_{\text {cnst }}}}\right) t
$$

where $s_{\mathrm{fr}}$ is the tangential (friction) stress; $s_{\mathrm{n}}$ is the normal stress; $\mu$ is the friction coefficient; $t$ is the tangential vector in the direction of the relative velocity; vr represents the relative sliding. Physically, the value of $r_{v_{\text {cnst }}}$ can be regarded as the value of the relative velocity below which sticking occurs.

In the simulation, the yield stress can be expressed by

$$
\sigma_{y}=C\left(\varepsilon_{0}+\bar{\varepsilon}\right)^{n}+D(\dot{\bar{\varepsilon}})^{k},
$$

where $\mathbf{e}_{0}$ is initial yield strain; $\bar{\varepsilon}$ is equivalent strain; $\dot{\bar{\varepsilon}}$ is equivalent strain rate; parameters $C, D, n$, and $k$ are material constants. Initially, the equivalent strain and equivalent strain rate were assumed to be zero so that the initial yield strain can be calculated by material constants $C$ and $n$ and Young's modulus $Y$ in von Mises material model. The von Mises stress is given by

$$
\bar{\sigma}=\frac{\left[\left(\sigma_{1}-\sigma_{2}\right)^{2}+\left(\sigma_{2}-\sigma_{3}\right)^{2}+\left(\sigma_{3}-\sigma_{1}\right)^{2}\right]^{1 / 2}}{\sqrt{2}},
$$

where $\mathbf{s}_{1}, \mathbf{s}_{2}$, and $\mathbf{s}_{3}$ are the principal Cauchy stresses along three main axes. The equivalent strain rate is

$$
\dot{\bar{\varepsilon}}=\left[\frac{2}{3}\left(\varepsilon_{\mathrm{ij}} \varepsilon_{\mathrm{ij}}\right)\right]^{1 / 2},
$$

where $\mathbf{e}_{i j}$ is the strain tensor.

MSC Marc uses Cauchy stress (true stress) and logarithmic strain with updated Lagrange formulation. It is instructive to derive the stiffness matrices for the updated Lagrangian formulation starting from the virtual work principle in the following equation:

$$
\int_{V_{0}} S_{\mathrm{ij}} \delta E_{\mathrm{ij}} \mathrm{d} V=\int_{V_{0}} b_{i}^{0} \delta \eta_{i} \mathrm{~d} V+\int_{A_{0}} t_{i}^{0} \delta \eta_{i} \mathrm{~d} A .
$$

Here, $S_{i j}$ is the second Piola-Kirchhoff stress; $E_{i j}$ is the Green-Lagrange strain; $b_{i}^{0}$ is the body force in the reference configuration; $t_{i}^{0}$ is the traction vector in the reference 
configuration; and $\eta \mathrm{i}$ is the virtual displacement. Direct linearization of the left-hand side of (5) yields

$$
\int_{V_{0}} S_{i j}\left(d\left(\delta E_{\mathrm{ij}}\right)\right) \mathrm{dV}=\int_{V_{n+1}} \nabla \eta_{\mathrm{ik}} \sigma_{\mathrm{kj}} \nabla \Delta u_{\mathrm{ij}} \mathrm{d} v,
$$

where $\Delta u$ and $\eta$ are actual incremental and virtual displacements, respectively, and $s_{\mathrm{kj}}$ is the Cauchy stress tensor. Equation (6) can be further calculated as

$$
\int_{V_{0}} \mathrm{~d} S_{i j} \delta E_{\mathrm{ij}} \mathrm{d} V=\int_{V_{n+1}} \nabla^{s} \eta_{\mathrm{ij}} L_{\mathrm{ijk} 1} \nabla^{s}\left(\Delta u_{k 1}\right) \mathrm{dv},
$$

where $\nabla^{S}$ denotes the symmetric part of $\nabla$, which represents the gradient operator in the present configuration. $L_{\mathrm{ijk} 1}$ is convected to the present configuration to yield:

$$
\left\{K_{1}+K_{2}\right\} \delta u=F-R,
$$

where $F$ and $R$ are the external and internal forces, respectively; $\delta u$ is the correction displacement vector; $K_{1}$ and $K_{2}$ are, respectively, the material stiffness matrix and geometric stiffness matrix, given by

$$
\left(K_{1}\right)_{\mathrm{ij}}=\int_{V_{n+1}} \beta_{\mathrm{imn}} L_{\mathrm{mnpq}} \beta_{\mathrm{pqj}} \mathrm{dv},
$$

and

$$
\left(K_{2}\right)_{\mathrm{ij}}=\int_{V_{n+1}} \sigma_{k 1} N_{\mathrm{i}, \mathrm{k}} N_{j, 1} \mathrm{dv},
$$

where $\beta_{\text {imn }}$ is the symmetric gradient operator evaluated in the present configuration; $L_{\text {mnpq }}$ represents the material moduli tensor; $s_{\mathrm{k} 1}$ is the Cauchy stresses; $N_{\mathrm{i}, \mathrm{k}}$ is the shape function gradient matrix.

Keeping in view that the reference state is the present state, a rate formulation can be expressed as

$\int_{V_{0}}\left[\dot{S}_{\mathrm{ij}} \delta E_{\mathrm{ij}}+S_{\mathrm{ij}} \frac{\partial v_{k}}{\partial X_{i}} \frac{\partial \delta \eta_{k}}{\partial X_{j}}\right] \mathrm{d} V=\int_{V_{0}} \dot{b}_{i} \delta \eta_{\mathrm{i}} \mathrm{d} V+\int_{A_{0}} \dot{t}_{i} \delta \eta_{i} \mathrm{dA}$,

where $\dot{S}_{\mathrm{ij}}$ is the rate of the second Piola-Kirchhoff stress. Equation (11) can be obtained by setting

$$
\begin{aligned}
F_{\mathrm{ij}} & =\delta_{\mathrm{ij}}, \\
\delta E_{\mathrm{ij}} & =\delta d_{\mathrm{ij}}, \\
\frac{\partial}{\partial X_{i}} & =\frac{\partial}{\partial x_{i}}, \\
S_{\mathrm{ij}} & =\sigma_{\mathrm{ij}},
\end{aligned}
$$

where $F_{\mathrm{ij}}$ is the deformation tensor; $d_{\mathrm{ij}}$ is the rate of deformation; $X_{\mathrm{i}}$ is the coordinate of initial node; and $x_{\mathrm{i}}$ is the coordinate of the node. Hence:

$$
\int_{V_{n+1}}\left[\underset{\sigma_{\mathrm{ij}}}{\nabla} \delta d_{\mathrm{ij}}+\sigma_{\mathrm{ij}} \frac{\partial v_{k}}{\partial x_{i}} \frac{\partial \delta \eta_{k}}{\partial x_{j}}\right] \mathrm{d} v=\int_{V_{n+1}} \dot{b_{i}} \delta \eta_{i} \mathrm{~d} v+\int_{V_{n+1}} \dot{t}_{i} \delta \eta_{i} \mathrm{da},
$$

in which $\dot{b}_{i}$ and $\dot{t}_{i}$ are the body force and surface traction in the present configuration, respectively. And in this equation, $\stackrel{\nabla}{\sigma_{\mathrm{ij}}}$ is the Truesdell rate of Cauchy stress.

MSC Marc uses Cauchy stress (true stress) and logarithmic strain with updated Lagrange formulation (explained in more detail in the preliminary work $[44,45]$ ).

2.2. Simulation Conditions. The boundary conditions of the MPFEM simulation are given as follows: displacement boundary condition is imposed via rigid upper punch; the positions of the rigid die wall and the lower punch are fixed; the change in density of the compact is determined by the change in displacement of the upper punch. The downward motion of the upper punch results in a downward force on the particles in contact with it, which in turn impart this force to their neighboring particles in the same direction. This process continues until the forces reach the die wall and the lower punch. It should be noted that the simulation results are not affected by the strain rate since materials are assumed to be strain rate insensitive. Each particle in the composite powder is independent and deformable; the relationship between the positions of particles can be determined in a contact table to judge their contact state in model setup. Upon compaction, the contact between particles was controlled by 'node to segment method' which is widely used in FEM simulation. The materials are set to be elastoplastic. Materials parameters for soft and hard particles used in the simulation are listed in Table 1 . The friction coefficient between particles is set to be 0.2 according to the Coulomb friction model being used. The punches and the die are rigid with smooth surface to reduce their friction with the powder. Once all the simulation parameters are determined, the simulation is run on the server.

\section{Results and Discussion}

3.1. Compaction Process and Macroscopic Analyses. In the forming process, the influence of the initial packing structure of the powder was considered in its entirety. In addition to the initial random packing before compaction, in order to increase the loose density of the composite powder, mechanical vibration to the composite powder in the DEM simulation was applied. The forming process of these powders was studied by numerical simulation and physical experiment, as shown in Figure 2. It can be seen that the results of physical experiment and numerical simulation are in agreement, which proves the effectiveness of the model used in our numerical simulation. In addition, we can also find that the change law of the three-dimensional compaction curve of the composite powder is similar to our previous two-dimensional simulation results $[40,44]$, and the specific reasons will not be described here. It should be mentioned that, during the formation of both soft and hard particles, although the initial packing structure of powder has little effect on the relative density of final compacts, it can be seen from Figure 2 that it has a significant influence on the early stage of forming process. At the same time, it also has a 


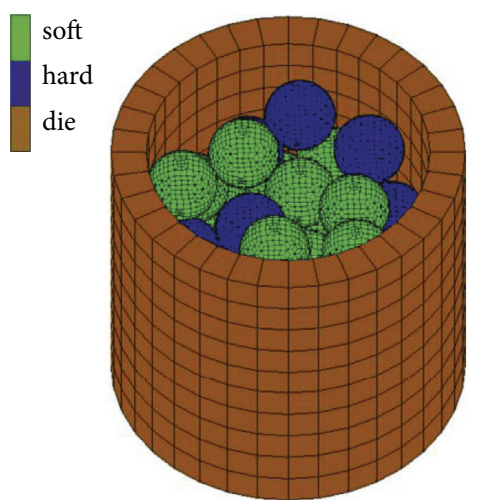

(a)

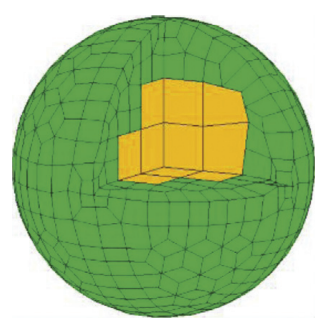

(b)

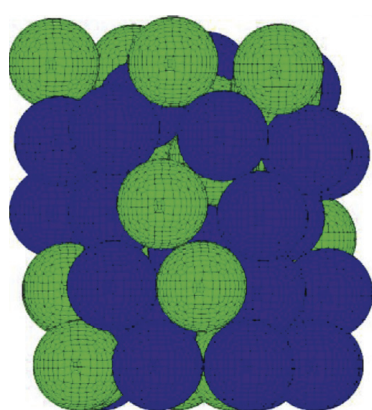

(c)

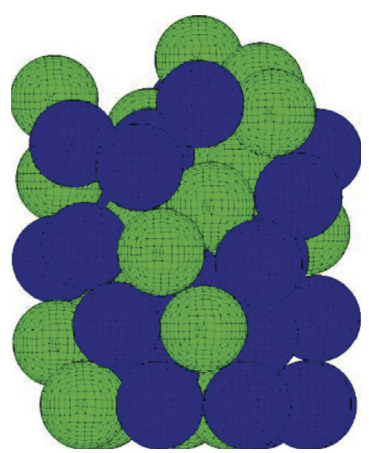

(d)

FIGURE 1: DEM generated 3D initial random dense packing structure (a) and mesh division of an individual particle in the soft and hard particles (b), the packing structures for with vibration (c) and (d) without vibration.

TABLE 1: Materials parameters of soft and hard particles used in the simulation.

\begin{tabular}{lcccc}
\hline Materials & Young's modulus Y/GPa & Poisson's ratio $v /-$ & Hardening index n/- & Strength coefficient C/MPa \\
\hline Soft & 62.59 & 0.33 & 0.25 & 225.9 \\
Hard & 120 & 0.25 & 0.237 & 575.3 \\
\hline
\end{tabular}

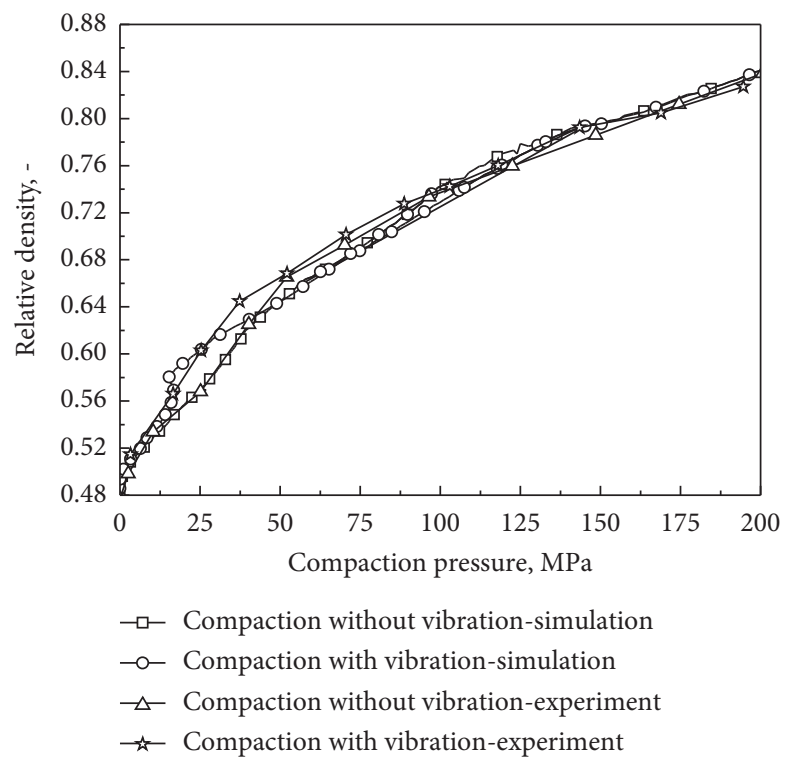

Figure 2: Numerical and physical compaction curves for the compaction of initial packing structures with and without mechanical vibration.

significant impact on the microproperties of the compaction, which will be discussed in the following sections.

In contrast to the macroscale continuous simulation method, our MPFEM simulation can also characterize the morphology and internal stress of the compact during the whole compaction process from the particle scale, as shown in Figure 3. In this case, the soft particles content in the composite powder is $25 \mathrm{wt} \%$; vibration treatment is applied to the powder before compaction. It can be seen from the figure that, in contrast to the two-dimensional compaction process, the evolution process of yield surface can be clearly observed in the three-dimensional compaction process of composite particles. This means that the mechanism of powder compaction densification can be accurately simulated from the microscopic point of view. As the compaction process evolves, it is found that the deformations of both soft and hard particles in the compaction gradually increase. In particular, the deformation of the soft particles is greater than that of the hard particles, and the stress concentration is mainly generated in the hard particles, which is similar to the stress concentration characteristics in two-dimensional compaction process [36]. It should be noted that the 


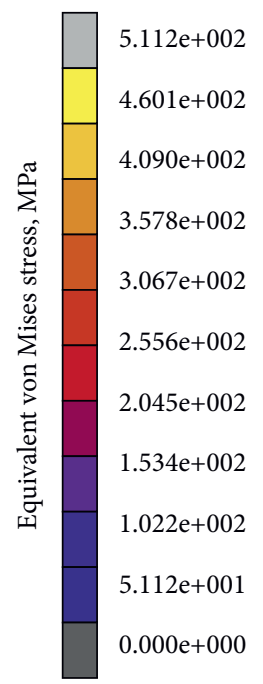

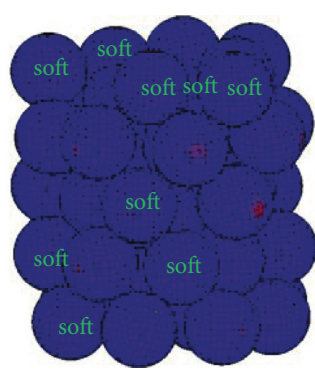

(a)

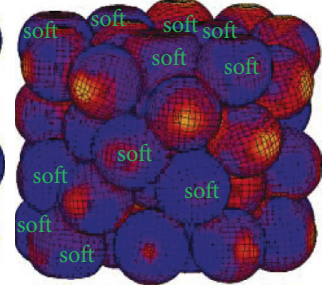

(b)

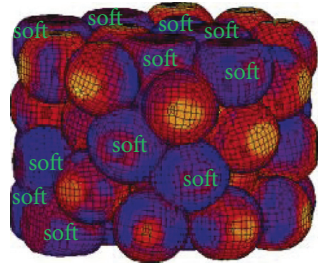

(c)

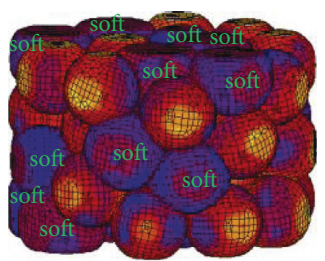

(d)

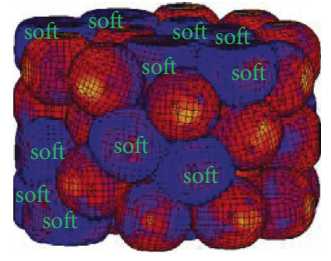

(e)

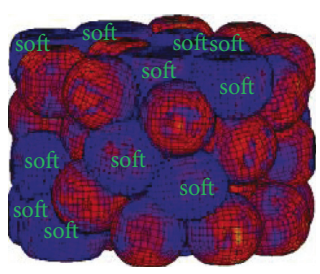

(f)

FIGURE 3: Evolution of the morphology and internal equivalent von Mises stress distribution of composite compact from loading to pressure releasing with mechanical vibration when $(\mathrm{X})_{\text {soft }}=25 \mathrm{wt} \%$. (a)-(f) correspond to $(\mathrm{P})=7,50,100$, and $200 \mathrm{MPa}$, pressure holding, and pressure releasing, respectively.

deformation of soft and hard particles gradually changes from curved surface to plane at the part contacting with the die wall, where the deformation amount of the two particles is almost equal, although the stress concentration of the deformation part of hard particles is still high. However, due to the release of internal stress after the pressure is relieved, the residual stress of both soft and hard particles near the edge of the die wall is almost equal, except for the residual stress in the hard particles that is evenly distributed. In addition, the size is almost equal; however, only the soft particles are under higher residual stress in the deformed part. This phenomenon is difficult to reflect in the two-dimensional forming process of composite powder [46, 47].

In order to further explore the mechanism that governs the filling of internal pores of the compacts and the evolution of the particle morphology during the three-dimensional compaction of composite powders, the compaction obtained under the condition of pressure $P=200 \mathrm{MPa}$ was layered and displayed, and the compaction morphology of different heights and the corresponding stress distribution were obtained as shown in Figure 4. It can be seen from the figure that the density at the bottom of the compact is lower than that at the top, which is caused by the top-down unidirectional die pressing used in this study. Since the force is transmitted from top to bottom through the upper die punch, the powder near the upper die punch is subjected to greater pressure and the degree of deformation during the forming process is relatively high. However, this deformation is not uniform. Usually, the pores in the contact parts of soft particles are filled more than the pores in the contact parts of hard particles. This conclusion is in good agreement with the maximum and minimum density distribution at the top edge and bottom edge of the compact obtained by continuous macroscopic simulation [20,22]. In addition, it can be seen from the figure that the deformation of soft particles is far greater than that of hard particles, and the closer the soft particles are to the upper die, the greater the deformation of soft particles. In addition, the surface is flatter, and the deformation of the lower soft particles is mainly caused by the action of hard particles. The surface of the soft particle that is in contact with the hard particle is inwardly curved. The irregular particle shape produced by this depression is difficult to squeeze into the adjacent pores inside the compact, which hinders further filling of the pores.

\subsection{Microscopic Property Characterization and Densification} Mechanism Analysis. In addition to the macroscopic properties, we also characterized and compared the microscopic properties of the composite powder during the forming process from the particle scale. Figure 5 shows the morphology and stress changes of single hard particle and contact area of soft particle in the center of the compaction at different stages of the pressing process of both soft and hard particles, where different colors of particles represent stress levels. Three states in the process of compaction were selected for study, and the relative densities of the compaction were $0.64,0.74$, and 0.84 , respectively. As can be seen from the figure, with the increase of pressing pressure, hard particles barely deform, but their color changes are manifest, which indicates that hard particles contact with other particles more and more closely, and the interaction between particles is also increasingly intense. On the other hand, the soft particles underwent large deformations, and the parts with significant deformation even exhibit inward depression, while the parts with bulges show almost no color change. This indicates that the part has not been in contact with other particles during the forming process or that the force in the contact area is very small even when it is in contact. The filling of pores is accomplished by the mass transfer at the protrusion site of soft particles. The stress at 

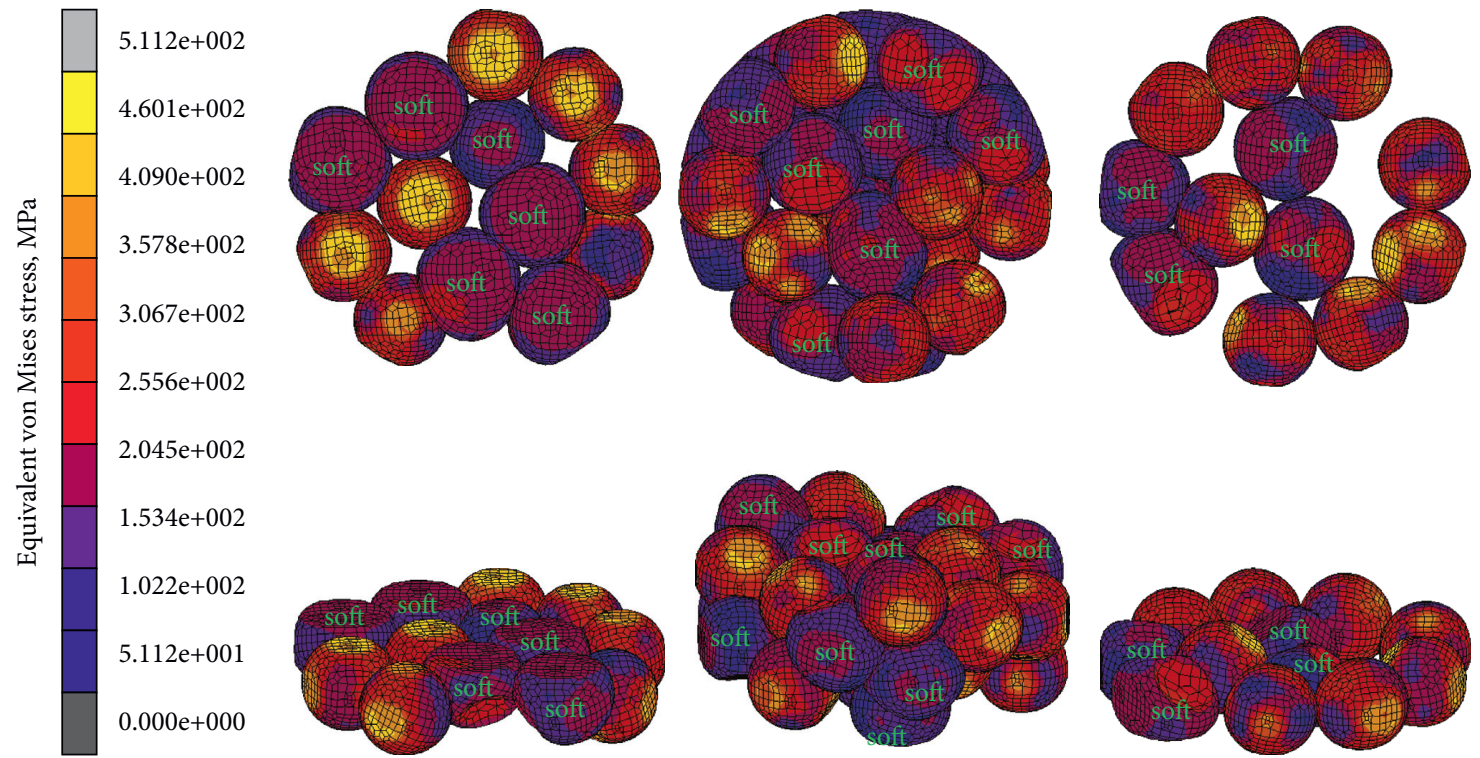

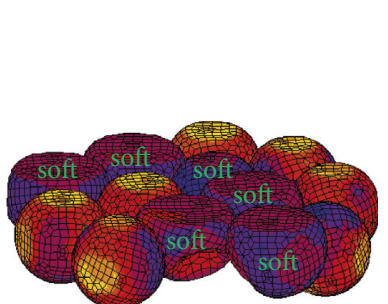

(a)

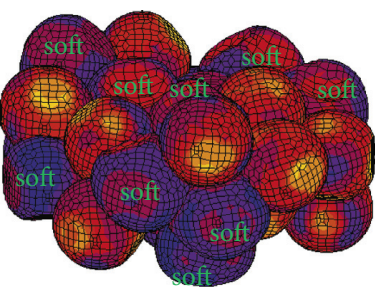

(b)

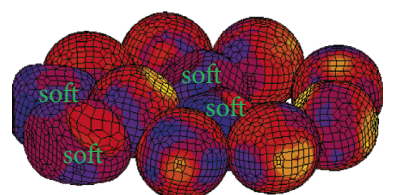

(c)

FIGURE 4: Equivalent von Mises stress distributions for local structures obtained from different heights of the compact when $(\mathrm{P})=200 \mathrm{MPa}$. (a)-(c) correspond to upper part, middle part, and bottom part of the compact.

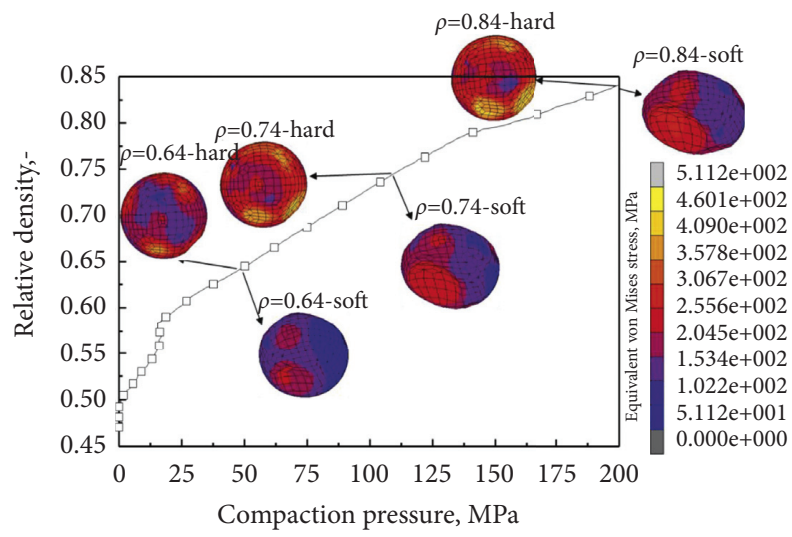

Figure 5: Evolution of the morphology of individual particles at different stages of compaction of vibrated initial packing structure of composite powder.

the deformation site where soft particles are in close contact with other particles is almost equal. The specific changes and their causes will be discussed systematically in the next part of the quantitative analysis. In addition, the $3 \mathrm{D}$ simulation results obtained here are similar to the $2 \mathrm{D}$ simulation results [44].

In order to further quantitatively characterize the deformation and mechanical behavior of the powder particles in the forming process, both soft and hard particles presented in Figure 4 were studied in depth, focusing on the distribution of internal stress in the two different particles and the evolution of residual stress after unloading as shown in Figure 6. As can be seen from the figure, the stress value in the center of hard particles in the compacted blank formed at $P=200 \mathrm{MPa}$ is far less than that at the surface with a large deformation, which is consistent with the two-dimensional simulation results. As can be seen from Figure 6, the locations with comparatively large particle shape variable correspond to locations where other hard particles contact each other. Since the yield strength of hard particles is higher than that of soft particles, the plastic deformation of hard particles requires more external energy, which causes greater concentration of stress at the plastic deformation site. However, for the soft particles in contact with it, the stress generated in the central part is not significantly different from the part with larger plastic deformation. There are two kinds of local deformation morphology of soft particles; one tends to be flat, and the other is concave to the inside of soft particles. The morphology that tends to be flat indicates that it is situated at the contact of two soft particles. As the pressing progresses, the contact between the two particles changes from point to surface. The inwardly concave morphology of soft particles indicates that the contact is between soft and hard particles. Due to the high hardness of 

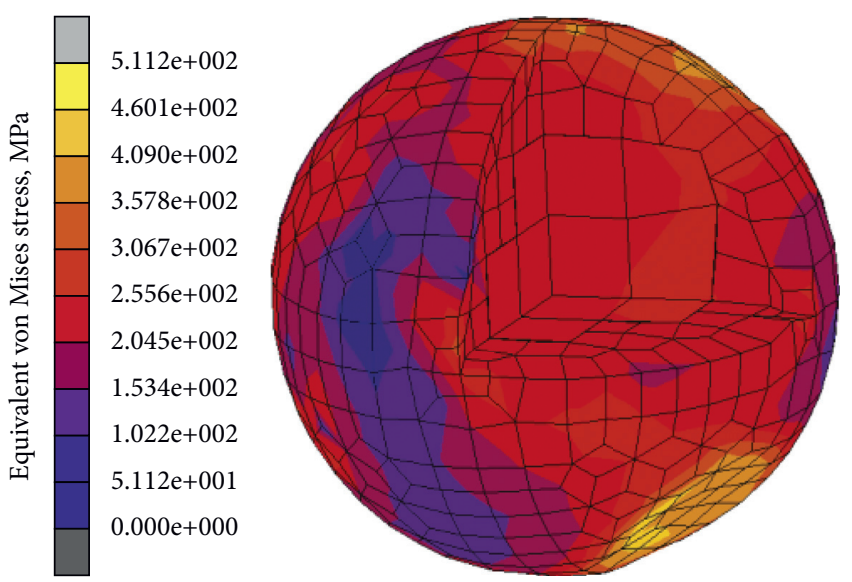

(a)

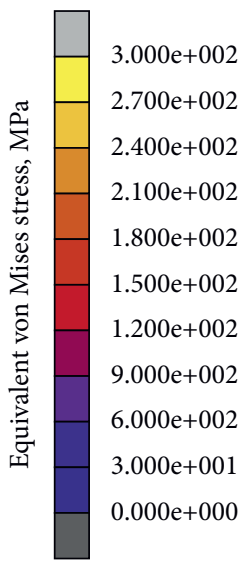

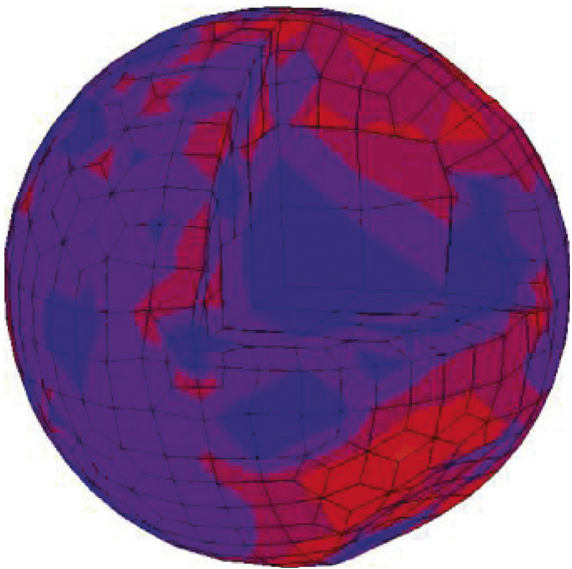

(b)

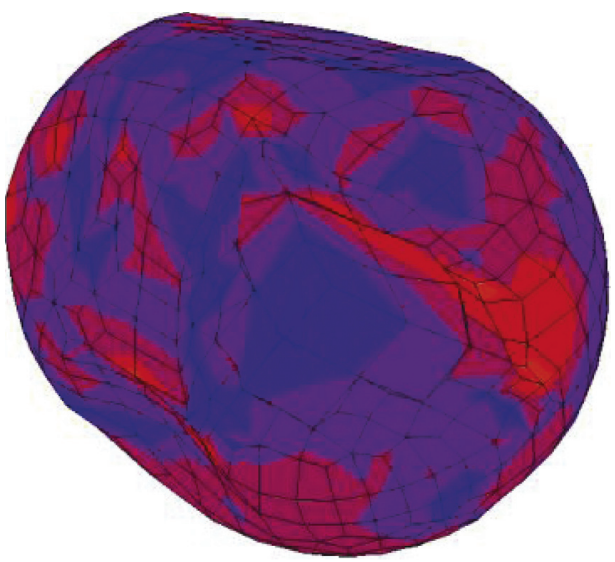

(d)

Figure 6: Equivalent von Mises stress distributions and morphology evolution of particles in Figure 5 at $(\mathrm{P})=200 \mathrm{MPa}$ and unloading. (a) and (b) show a hard particle and (c) and (d) show a soft particle; left figures correspond to $(\mathrm{P})=200 \mathrm{MPa}$ and right to unloading.

hard particles, the deformation of hard particles is very small during the forming process. Under the action of the hard particles, soft particles exhibit large plastic deformation and can envelop the hard particles. In this case, the maximum stress is concentrated in the depression of soft particles. After the pressure is relieved, the internal stress of the particles is redistributed and the self-equilibrium of stress is achieved. It should be noted that the stress in the center of both soft and hard particles is reduced almost to zero, and comparatively high residual stress remains only at the edges and corners of the particles.

The stress distribution inside hard particles in Figure 6 was quantitatively characterized. Figure 7 (a) shows the equivalent von Mises stress distribution inside hard particles along three axes from the center $(0.5 \mathrm{~mm})$. It can be seen from the figure that the equivalent von Mises stress value of the hard particle varies from $175 \mathrm{MPa}$ to $340 \mathrm{MPa}$ and the distribution is not uniform. The stress value in the center of the particle is about $230 \mathrm{MPa}$, and it fluctuates greatly in the process of extending to the edge of the particle. However, regardless of the axis of direction, greater equivalent von Mises stress values are found closer to the edge of the particle. In the process of unidirectional die pressing, the upper die moves along the negative direction of $Z$ axis, and the maximum stress on the upper surface of hard particles is about $340 \mathrm{MPa}$. Figure 7 (b) shows the distribution of equivalent von Mises stress in the center of hard particle $(0.5 \mathrm{~mm})$ along three axes after pressure relief. It can be seen that the equivalent von Mises stress in the center of the hard particles drops from $230 \mathrm{MPa}$ to $80 \mathrm{MPa}$ after the pressure is relieved, and the distribution from the center to both sides is more uneven. The equivalent von Mises stress value varied from $60 \mathrm{MPa}$ to $250 \mathrm{MPa}$. Although it decreased after pressure relief, the difference between maximum and minimum is very close the equivalent difference inside the particle at $P=200 \mathrm{MPa}$. The larger internal stress difference is not conducive to the subsequent sintering process.

In a similar fashion, we also quantitatively characterized the internal stress distribution of the soft particles in Figure 6. Figure 8(a) shows the equivalent von Mises stress distribution from the center $(0.5 \mathrm{~mm})$ of the soft particles along the three coordinate axes. It can be seen from the figure that the equivalent von Mises stress value of the soft particles varies between $137 \mathrm{MPa}$ and $240 \mathrm{MPa}$, and the internal stress distribution is more uneven than that of the hard particles in Figure 7(a). This is because the deformation 


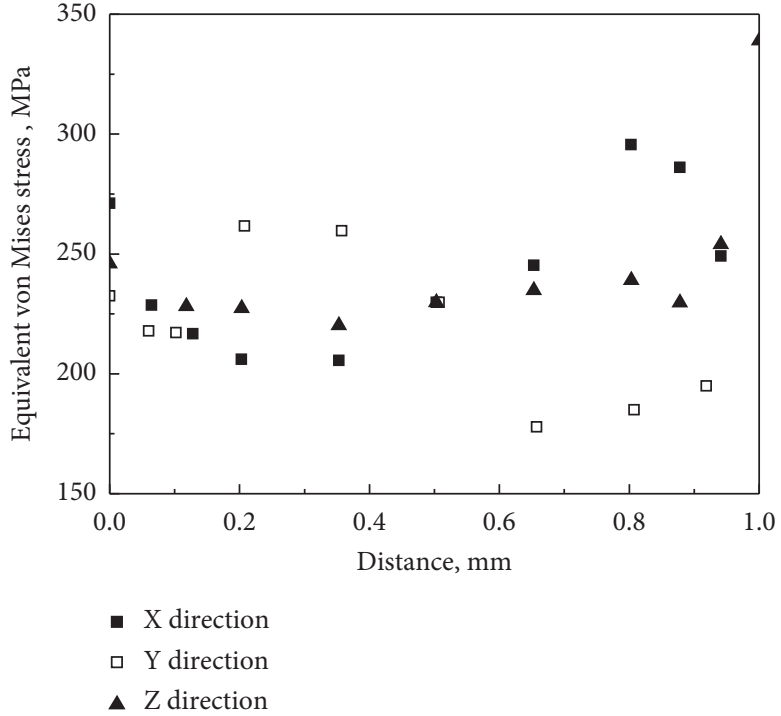

(a)

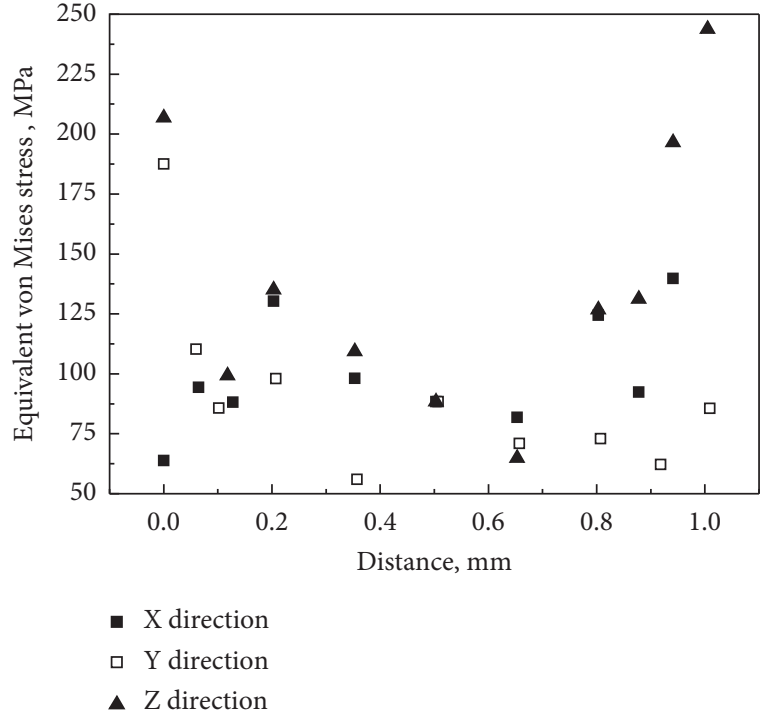

(b)

Figure 7: Equivalent von Mises stress distributions of hard particle (from center $(0.5 \mathrm{~mm})$ to outside) along (X) $Y$ and $Z$ directions. (a) $P=200 \mathrm{MPa}$; (b) unloading.

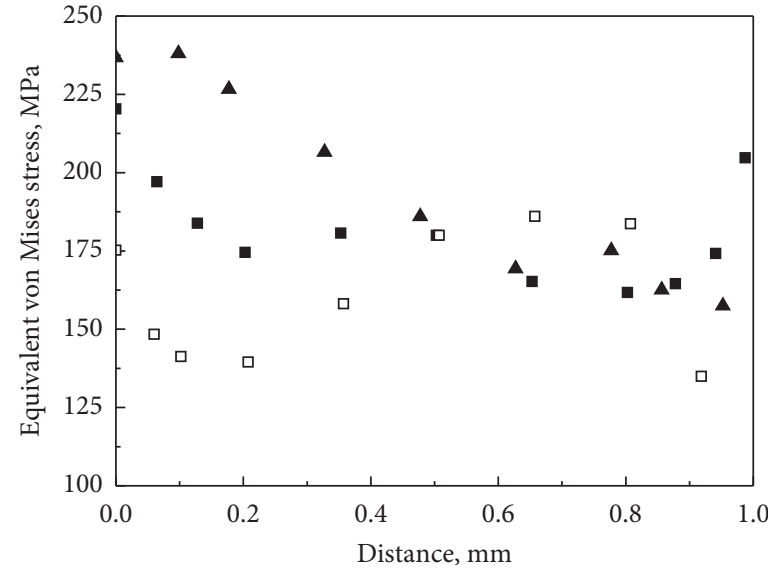

- X direction

口 $\mathrm{Y}$ direction

- Z direction

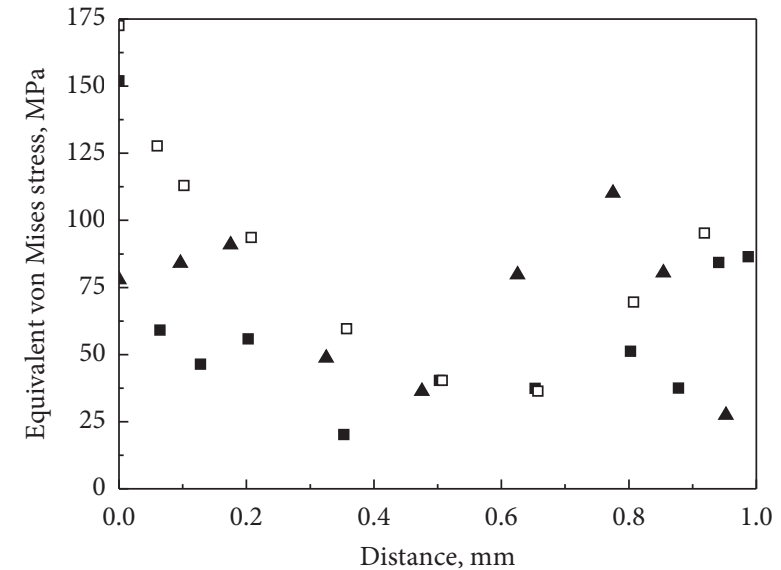

- X direction

口 Y direction

- Z direction

(a)

(b)

Figure 8: Equivalent von Mises stress distributions in soft particle from center $(0.5 \mathrm{~mm})$ to exterior along (X) $Y$ and $Z$ directions. (a) $(\mathrm{P})=200 \mathrm{MPa}$, (b) unloading.

of the soft particles is greater than that of the hard particles during the forming process, which generates multiple shear forces inside the soft particles. The stress value in the center of soft particles is about $175 \mathrm{MPa}$, which is $55 \mathrm{MPa}$ lower than that in the center of hard particles. Although the upper die moves along the negative direction of the $Z$ axis, the equivalent von Mises stress at the lower part of the particle is the highest. This is because the lower part of the particle is influenced by the action of hard particles (the most severe part of the depression in Figure 5 is the lower part of the soft particle), and the maximum stress on the lower surface of the particle is about $240 \mathrm{MPa}$. Figure 8 (b) shows the distribution of equivalent von Mises stress along three axes from the soft particle center $(0.5 \mathrm{~mm})$ after pressure relief. By comparing the equivalent von Mises stress distribution within hard particles after pressure relief (Figure 7(b)), it can be seen that the stress distribution within soft particles is more uneven. However, the equivalent von Mises stress value within soft particles varies between $25 \mathrm{MPa}$ and $175 \mathrm{MPa}$, and the difference is smaller than that within hard particles after pressure relief. Therefore, in order to obtain high quality compaction, we should devote ourselves to relieving the 


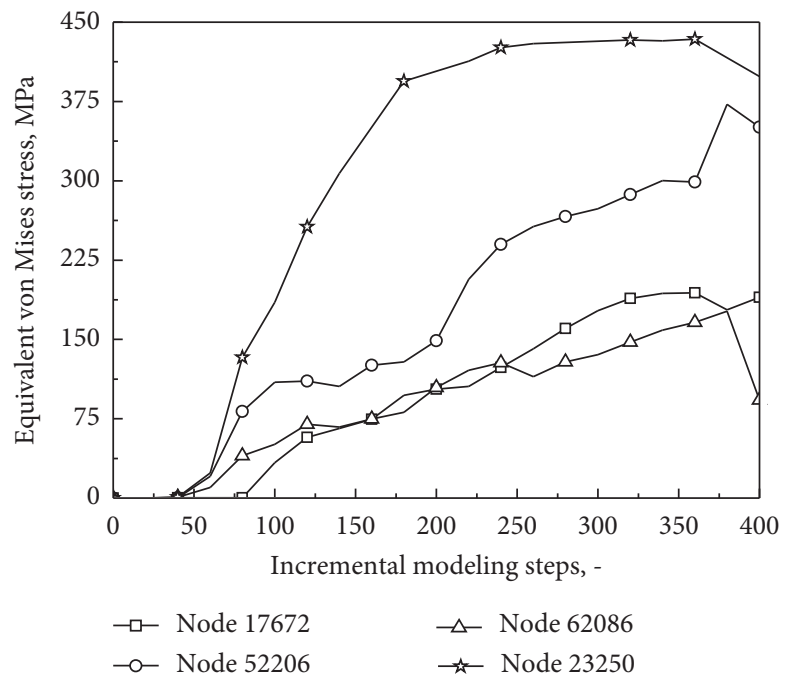

Figure 9: Variation of equivalent von Mises stress with incremental modelling step for different particle nodes in the compact during compaction.

larger stress gradient inside the particle and reducing the stress gradient inside the particle.

In order to further study the characteristics of the maximum stress generated on the surface of both soft and hard particles, the ones in the center of the compaction were considered as the objects, and the points producing the maximum stress on their surfaces were selected, among which the node on soft particle was Node17672 and the node on hard particle was Node52206. In addition, both soft and hard particles near the die wall were also considered as objects, and the points producing the maximum stress value on their surfaces were selected, among which there is the node Node62086 on soft particle and the node Node23250 on hard particle. The stress present at these nodes was compared and analyzed. It should be noted that these points represent the maximum stress points in the compaction, and the selected particles are the center of compaction and close to the die wall, in order to be representative and universal. The results are shown in Figure 9. As can be seen from the figure, the equivalent von Mises stress values at these nodes increase continuously with the process of formation. However, the equivalent von Mises stress values of these nodes decrease after pressure relief. By contrast, the equivalent von Mises stress value at soft particle nodes is much smaller than that at hard particle nodes, and the difference becomes larger with the increase of the number of simulated steps. The maximum difference of equivalent von Mises stress between soft and hard particles in the center at the same number of simulation steps is about $200 \mathrm{MPa}$, and the maximum difference near the die wall with the same number of simulation steps is about $300 \mathrm{MPa}$. However, the equivalent von Mises stress values for the soft particles in the center and near the die wall are not significantly different, while the equivalent von Mises stress values of the hard particles in the center and near the die wall are significantly different with the maximum difference of $220 \mathrm{MPa}$. This is because the deformation of soft particles in any position is very large during the forming process, while the hard particles near the die wall exhibit greater deformation than in the center. After the pressure is relieved, the equivalent von Mises stress value of the particle near the die wall decreased more than that of the particle at the center. However, the residual stress at the particles near the die wall remains comparatively large, node17672 about $187 \mathrm{MPa}$; node52206 about $337 \mathrm{MPa}$; node62086 about $80 \mathrm{MPa}$; node23250 about $385 \mathrm{MPa}$.

\section{Conclusions}

In this paper, the MPFEM method was used to simulate the three-dimensional forming process of composite powder at the particle scale. The effect of the initial packing structure of the powder before compaction was investigated. The macroscopic and microscopic properties of the composite powders during the compaction process were quantitatively characterized and analyzed. In addition, the densification mechanism of the composite powders was studied from the perspective of pore filling and force. The main conclusions obtained are as follows:

(1) In the 3D numerical simulation of the forming process of composite powders, the process of pressing after vibration is applied to the powder before forming can result in higher relative density under low pressure. The vibration can result in more favorable arrangement of the particles and eliminate the bridge and arch structures that hinder the uniform distribution of pores in the initial packing structure.

(2) The $3 \mathrm{D}$ numerical simulation can reproduce the pore filling mechanism of the composite powder compaction, evolution of the internal stress, and particle morphology of the composite powder compact in 
real time. After vibration is applied to the powder before forming, the distribution of pores in the compacted blank after forming is more uniform than that without vibration, and the pores close to the upper die part of the compacted blank are smaller than those in the lower part of the compacted compact. The surface deformation of the particles near the die wall and the upper and lower parts of the punching die is relatively smooth, while the interior particles, particularly the soft particles, exhibit concave surface due to the extrusion of hard particles. The maximum stress occurs at the edges and corners of deformation and in the center of plane and concave with severe deformation.

(3) For either soft or hard particles, the stress is concentrated at their central part during the process of pressing, and the internal stress distribution is not uniform. After the pressure is relieved, the stress concentration in the center of the particle is greatly reduced, and the stress is rebalanced. The larger stress concentration appears at the edges and corners of the deformed particle, which causes the internal stress distribution of the particle after the pressure is relieved to be extremely uneven. In contrast, the stress inhomogeneity inside soft particles is higher than that inside hard particles. In the subsequent sintering process, cracks occur not only at the contact points of the particles, but also at the edges and corners of the particles, resulting in a decrease in the quality of sintered compact.

(4) The results of the physical experiment are in good agreement with the numerical simulation, which proves the validity of the numerical model used in the MPFEM simulation. The results of physical experiments show that applying vibration to the powder before forming can increase the loose packing density of the composite powder, and simultaneously time improves both macro- and microproperties of the compact.

\section{Data Availability}

The data used to support the findings of this study are included within the article.

\section{Conflicts of Interest}

The authors declare that there are no conflicts of interest regarding the publication of this paper.

\section{Acknowledgments}

The authors are grateful to the financial support by Education Department Project of Liaoning Province (2020LNQN12), National Key R\&D Project of China (2017YFC0805100), and Fundamental Research Funds for University of Science and Technology Liaoning (2018QN13) for the financial support of current work.

\section{References}

[1] K. Yamaguchi, N. Takamura, and S. Imatani, "Compaction and sintering characteristics of composite metal powders," Journal of Materials Processing Technology, vol. 63, pp. 1-3, 1997.

[2] A. H. Tavakoli, A. Simchi, and S. M. S. Reihani, "Study of the compaction behavior of composite powders under monotonic and cyclic loading," Composites Science and Technology, vol. 65, no. 14, pp. 2094-2104, 2005.

[3] K. T. Kim, S. C. Lee, and H. S. Ryu, "Densification behavior of aluminum alloy powder mixed with zirconia powder inclusion under cold compaction," Materials Science and Engineering: $A$, vol. 340, no. 1-2, pp. 41-48, 2003.

[4] C. A. Leon, G. O. Rodriguez, and E. A. R. Aguilar, "Cold compaction of metal-ceramic powders in the preparation of copper base hybrid materials," Materials Science and Engineering: $A$, vol. 526, no. 1-2, pp. 106-112, 2009.

[5] M. F. Moreno and C. J. R. O. González, "Densification of Al powder and $\mathrm{Al}-\mathrm{Cu}$ matrix composite (reinforcedwith15\% Saffilshortfibres) during axial cold compaction," Powder Technology, vol. 206, pp. 297-305, 2011.

[6] D. F. Khan, H. Yin, H. Li et al., "Compaction of Ti-6Al-4V powder using high velocity compaction technique," Materials \& Design, vol. 50, pp. 479-483, 2013.

[7] A. R. Khoei, A. R. Azami, and S. Azizi, "Computational modeling of 3D powder compaction processes," Journal of Materials Processing Technology, vol. 185, no. 3, pp. 166-172, 2007.

[8] K. Biswas, "Comparison of various plasticity models for metal powder compaction processes," Journal of Materials Processing Technology, vol. 166, no. 1, pp. 107-115, 2005.

[9] S. M. Tahir, A. K. Ariffin, and M. S. Anuar, "Finite element modelling of crack propagation in metal powder compaction using Mohr-Coulomb and Elliptical Cap yield criteria," Advanced Powder Technology, vol. 202, no. 1, pp. 162-170, 2010.

[10] H. Diarra, V. Mazel, V. Busignies, and P. Tchoreloff, "FEM simulation of the die compaction of pharmaceutical products: influence of visco-elastic phenomena and comparison with Experiments," International Journal of Pharmaceutics, vol. 453, no. 2, pp. 389-394, 2013.

[11] A. K. Eksi and A. H. Yuzbasioglu, "Effect of sintering and pressing parameters on the densification of cold isostatically pressed Al and Fe powders," Materials \& Design, vol. 28, no. 4, pp. 1364-1368, 2007.

[12] K. A. Kuhn and C. L. Downey, "Deformation characteristics and plasticity theory of sintered powder materials," International Journal of Powder Metallurgy, vol. 7, no. 1, pp. 15-25, 1971.

[13] R. J. Green, "A plasticity theory for porous solids," International Journal of Mechanical Sciences, vol. 14, no. 3, pp. 215-224, 1972.

[14] S. Shima and M. Oyane, "Plasticity theory for porous metallurgy," International Journal of Mechanical Sciences, vol. 18, no. 6, pp. 285-291, 1971.

[15] A. L. Gurson, "Continuum theory of ductile rupture by void nucleation and growth: Part I-Yield criteria and flow rules for porous ductile media," Journal of Engineering Materials and Technology, vol. 99, no. 1, pp. 2-15, 1977.

[16] S. M. Doraivelu, H. L. Gegel, and J. S. Gunasekera, "A new yield function for compressible materials," International Journal of Mechanical Sciences, vol. 26, no. 9, pp. 527-535, 1984.

[17] D. C. Drucker and W. Prager, "Soil mechanics and plastic analysis or limit design," Quarterly of Applied Mathematics, vol. 10, no. 2, pp. 157-165, 1952. 
[18] K. H. Roscoe, A. N. Schofield, and C. P. Wroth, "On the yielding of soils," Géotechnique, vol. 8, no. 1, pp. 22-53, 1958.

[19] X. An, Z. Xing, and C. Jia, "Cold compaction of copper powders under mechanical vibration and uniaxial compression," Metallurgical and Materials Transactions A, vol. 45, no. 4, pp. 2171-2179, 2014.

[20] X. An, Y. Zhang, Y. Zhang, and S. Yang, "Finite element modeling on the compaction of copper powder under different conditions," Metallurgical and Materials Transactions A, vol. 46, no. 8, pp. 3744-3752, 2015.

[21] Y. X. Zhang, X. Z. An, and Y. L. Zhang, "Multi-particle FEM modeling on microscopic behavior of $2 \mathrm{D}$ particle compaction," Applied Physics A, vol. 118, no. 3, pp. 1015-1021, 2015.

[22] P. Han, X. Z. An, Y. X. Zhang, and Z. S. Zou, "FEM modeling on the compaction of $\mathrm{Fe}$ and $\mathrm{Al}$ composite powders," Journal of Mining and Metallurgy, Section B: Metallurgy, vol. 51, no. 2, pp. 163-171, 2015.

[23] F. Huang, X. An, Y. Zhang, and A. B. Yu, "Multi-particle FEM simulation of $2 \mathrm{D}$ compaction on binary $\mathrm{Al} / \mathrm{SiC}$ composite powders," Powder Technology, vol. 314, pp. 39-48, 2017.

[24] X. Z. An, R. Y. Yang, K. J. Dong, R. P. Zou, and A. B. Yu, "Micromechanical simulation and analysis of one-dimensional vibratory sphere packing," Physical Review Letters, vol. 95, no. 20, Article ID 205502, 2005.

[25] A. B. Yu, X. Z. An, R. P. Zou, R. Y. Yang, and K. Kendall, "Selfassembly of particles for densest packing by mechanical vibration," Physical Review Letters, vol. 97, no. 26, Article ID 265501, 2006.

[26] Y. L. Wu, X. Z. An, and A. B. Yu, "DEM simulation of cubical particle packing under mechanical vibration," Powder Technology, vol. 314, pp. 89-101, 2016.

[27] B. Zhao, X. An, Y. Wang, Q. Qian, and X. Yang, "DEM dynamic simulation of tetrahedral particle packing under $3 \mathrm{D}$ mechanical vibration," Powder Technology, vol. 317, pp. 171-180, 2017.

[28] C. L. Martin and D. Bouvard, "Isostatic compaction of bimodal powder mixtures and composites," International Journal of Mechanical Sciences, vol. 46, no. 6, pp. 907-927, 2004.

[29] C. PavanaChand and R. KrishnaKumar, "Yield function for metal powder compaction based on micromechanics of particle deformation," Scripta Materialia, vol. 35, no. 6, pp. 767-774, 1996.

[30] R. S. Ransing, D. T. Gethin, A. R. Khoei, P. Mosbah, and R. W. Lewis, "Powder compaction modelling via the discrete and finite element method," Materials \& Design, vol. 21, no. 4, pp. 263-269, 2000.

[31] I. Sridhar, N. A. Fleck, and A. R. Akisanya, "Cold compaction of an array of cylindrical fibres," International Journal of Mechanical Sciences, vol. 43, no. 3, pp. 715-742, 2001.

[32] I. M. Cameron and D. T. Gethin, "Exploration of die wall friction for powder compaction using a discrete finite element modelling technique," Modelling and Simulation in Materials Science and Engineering, vol. 9, no. 4, pp. 289-307, 2001.

[33] X. J. Xin, P. Jayaraman, G. Jiang, R. H. Wagoner, and G. S. Daehn, "Explicit finite element method simulation of consolidation of monolithic and composite powders," Metallurgical and Materials Transactions A, vol. 33, no. 8, pp. 2649-2658, 2002.

[34] K. Mori and R. Kuzime, "Microscopic approach of powder compaction using finite element method," International Journal of Mechanical Sciences, vol. 44, no. 4, pp. 793-807, 2002.

[35] A. Zavaliangos, "A multiparticle simulation of powder compaction using finite element discretization of individual particles," MRS Proceedings, vol. 731, pp. 733-717, 2002.
[36] A. T. Procopio and A. Zavaliangos, "Simulation of multi-axial compaction of granular media from loose to high relative densities," Journal of the Mechanics and Physics of Solids, vol. 53, no. 7, pp. 1523-1551, 2005.

[37] K. H. Lee, J. M. Lee, and B. M. Kim, "Densification simulation of compacted $\mathrm{Al}$ powders using multi-particle finite element method," Transactions of Nonferrous Metals Society of China, vol. 19, no. s1, pp. 68-75, 2009.

[38] J.-F. Jerier, B. Hathong, V. Richefeu et al., "Study of cold powder compaction by using the discrete element method," Powder Technology, vol. 208, no. 2, pp. 537-541, 2011.

[39] B. Harthong, J.-F. Jérier, V. Richefeu et al., "Contact impingement in packings of elastic-plastic spheres, application to powder compaction," International Journal of Mechanical Sciences, vol. 61, no. 1, pp. 32-43, 2012.

[40] F. Güner, O. N. Cora, and H. Sofuoğlu, "Numerical modeling of cold powder compaction using multi particle and continuum media approaches," Powder Technology, vol. 271, pp. 238-247, 2015.

[41] W. Wu, G. Jiang, R. H. Wagoner, and G. S. Daehn, "Experimental and numerical investigation of idealized consolidation," Acta Materialia, vol. 48, no. 17, pp. 4323-4330, 2000.

[42] J. Zhang, "A study of compaction of composite particles by multi-particle finite element method," Composites Science and Technology, vol. 69, no. 13, pp. 2048-2053, 2009.

[43] B. Harthong, F. Jérier, P. Dorémus, D. Imbault, and V. Donzé, "Modeling of high-density compaction of granular materials by the Discrete Element Method," International Journal of Solids and Structures, vol. 46, no. 18-19, pp. 3357-3364, 2009.

[44] B. Harthong, D. Imbault, and P. Dorémus, "The study of relations between loading history and yield surfaces in powder materials using discrete finite element simulations," Journal of the Mechanics and Physics of Solids, vol. 60, no. 4, pp. 784-801, 2012.

[45] P. Han, X. An, Y. Zhang et al., "Particulate scale MPFEM modeling on compaction of Fe and Al composite powders," Powder Technology, vol. 314, pp. 69-77, 2017.

[46] Y. Y. Foo, Y. Sheng, and B. J. Briscoe, "An experimental and numerical study of the compaction of alumina agglomerates," International Journal of Solids and Structures, vol. 41, no. 21, pp. 5929-5943, 2004.

[47] R. Zhou, H. Zhang, and Y. He, "Numerical simulation of residual stress field in green power metallurgy compacts by modified Drucker-Prager Cap model," Transactions of Nonferrous Metals Society of China, vol. 23, no. 8, pp. 2374-2382, 2013. 